\title{
Exercise-Based VRT Shows Benefits in Balance and Postural Stability in Adult Patients with Unilateral Vestibular Hypofunction
}

\author{
Kambiz Ahmadi Angali ${ }^{1}$, Arash Bayat ${ }^{2}$, Nader Saki $^{2}$ and Golshan Mirmomeni ${ }^{2, *}$ \\ ${ }^{1}$ Department of Biostatistics and Epidemiology, School of Public Health, Ahvaz Jundishapur University of \\ Medical Sciences, Ahvaz, Iran \\ ${ }^{2}$ Hearing Research Center, Imam Khomeini Hospital, Ahvaz Jundishapur University of Medical Sciences, \\ Ahvaz, Iran
}

\begin{abstract}
Background and Objective: Vestibular rehabilitation therapy (VRT) is an exercise-based program designed to improve balance function and alleviate problems related to dizziness, including vestibular hypofunction. This study aimed to evaluate the therapeutic effects of VRT exercises on balance function in adults with unilateral vestibular hypofunction (UVH).

Methods: Thirty-one patients (mean age: $39.48( \pm 10.96)$ years old) with confirmed UVH participated in this study. The VRT program consisted of habituation and adaptation exercises in combination with gait and balance exercises. The handicapping influences of dizziness were measured with a dizziness handicap inventory (DHI) scale to measure the level of respondent's performance on physical, emotional, and functional dimensions. Visual analogue scale (VAS) was also used to rate the severity of dizziness symptoms. The DHI and VAS scales were assessed pre-intervention and postintervention at one, two, and four weeks follow-ups after the last rehabilitation session.
\end{abstract}

Results: An analysis of variance showed a significant reduction and a consequent improvement in DHI scores after vestibular exercises in all domains: emotional, physical and functional $(p<0.001)$. The mean total DHI score significantly decreased by 15 points after one week, 24 points after two weeks and 15 points after four weeks of the last VRT session. The mean baseline VAS score was $7.71( \pm 0.91)$ that significantly decreased at one, two, and four weeks postintervention to $4.81( \pm 0.73), 3.77( \pm 0.61)$, and $3.16( \pm 0.97)$, respectively $(p<0.001)$.

Conclusion: The exercise-based VRT shows benefits for adult patients with chronic dizziness concerning improvement in the vertigo symptom scale, fall risk, balance and emotional status.

Keywords: Vestibular rehabilitation, Adult, Unilateral vestibular dysfunction.

\section{INTRODUCTION}

Vestibular hypofunction occurs when the balance system within the inner ear as the peripheral vestibular system does not properly function. The vestibular hypofunction could be unilateral or bilateral, engaging the one or both vestibular system located within the inner ears. Unilateral vestibular hypofunction (UVH) is a disturbance in the function of one side of the vestibular system in the inner ear that helps control balance and eye movements. The common symptoms in patients with UVH are vertigo, imbalance, lightheadedness, and oscillopsia. These patients may also display impaired spatial orientation, postural instability and gait disorders [1-5]. These impairments may lead to significant restrictions in the patient's daily activity and participation $[6,7]$.

Pharmacologic treatment and surgery are the two most commonly used approaches for the management of UVH. However, these approaches have limited

*Address correspondence to this author at the Hearing Research Center, Imam Khomeini Hospital, Ahvaz Jundishapur University of Medical Sciences, Ahvaz, Iran; Tel: 009863 3743102; Fax: 0098633743506 ;

E-mail: mirmomeni@gmail.com positive effect in these patients [7-10]. Vestibular rehabilitation therapy (VRT) consists of systematic, repetitive protocols aimed to facilitate central nervous system plasticity in patients with balance function problems [11-14]. Recently, different VRT protocols have been widely used as an effective option for the treatment of clinical symptoms and physical performances of patients with vestibular dysfunction, including UVH [15-18].

It has been shown that VRT exercises can alleviate the symptoms of dizziness, anxiety and depression, and improve static and dynamic balance, selfconfidence, and quality of life in patients with vestibular disturbances [19-23]. These exercises are predominantly based on graded activities including eye, head and body movements stimulating the vestibular system. VRT protocols usually consist of adaptation, substitution or habituation strategies. Habituation or compensatory exercises reduce dizziness symptoms through repetition of symptom-provoking head movements. Adaptation exercises including repeated head movements while focusing on a target, gaze stabilization helps the central nervous system by adapting to a loss or a change in vestibular system 
input. Finally, the substitution exercises organize the use of the remaining sensory inputs to aid postural control.

Giray et al. [24] in a randomized controlled trial assessed the short-term effects of VRT exercises on the symptom, disability, balance, and postural stability in adults with chronic unilateral vestibular dysfunction (UVD). They reported significant improvements in symptom, disability, balance, and postural stability in the affected patients following an exercise program indicating that customized exercise programs can be adapted to suit the specific needs of patients suffering from chronic UVD.

To our knowledge, so far, few studies have employed VRT for patients with UVH. Therefore, this prospective study was aimed to examine the efficacy of VRT on different symptoms of adult patients with UVH.

\section{METHODS}

\section{Participants}

A total of 31 patients (M/F: 16/15; mean age: 39.48 ( \pm 10.96$)$; range: 24 to 59 years) participated in this study. These patients were diagnosed as UVH $(>1$ year) at the department of otorhinolaryngology between August 2015 and September 2017. Exclusion criteria included a history of symptoms duration of fewer than 2 months, neurological disorder, cognitive impairment, post-traumatic vertigo, cervical problem, cerebrovascular diseases, and body balance rehabilitation in the last 6 months. The diagnosis of UVH was based on the positive clinical head impulse test and criterion of $>25 \%$ reduced vestibular responses on the caloric test during videonystagmography assessment (ICS Chartr, GN Otometrics, Denmark).

The protocols and all experimental procedures of the study were approved by the ethics committee of the local research committee (Registration code: IR.AJUMS.REC.1397.497), which were in complete agreement with the ethical regulations of human studies set by the Helsinki declaration (2013). After the enrolment of all subjects and before the start of the study, researchers completely and clearly explained all objectives and protocols of the study and possible benefits and side effects of the treatments to all participants, and then, all of the patients filled and signed a written consent form on their participation in the study.

\section{Experimental Procedures}

After taking a detailed case history, and performing a physical and vestibular function assessments, an exercise plan was developed for each patient. The VRT program consisted of habituation and adaptation exercises in combination with gait and balance exercises [13, 24, 25].

The VRT habituation exercises alleviate vertigo/dizziness through repetition of symptomprovoking head movements. For this reason, participants were instructed to fix gaze during ambulation to stabilize walking and to decrease veering to the side or to stand on the foam with a closed eye to keep their balance status.

During adaptation exercises, patients were initially asked to move their heads in yaw rotation while focusing on a stationary target, $X 1$ viewing. They then progressed to $\mathrm{X} 2$ viewing, in which the goal and the head rotated in equal and opposite yaw directions. These exercises were conducted in vertical and horizontal planes three times a day for one minute each.

All participants were asked to perform the exercises 4 to 5 times daily for a total of 20 to 30 minutes per day plus 20 minutes of balance and gait exercises.

The handicapping influences of dizziness were measured using a dizziness handicap inventory (DHI) scale. This scale consisted of 25 items that quantify the level of respondent's performance on physical ( 7 items), emotional (9 items), and functional (9 items) subscales. The DHI score for each item is calculated based on the subject's answer: no (0 points), sometimes ( 2 points), or yes (4 points). The DHI scored from 0 to 100 points with higher scores indicating more handicap [26]. The DHI scores are also classified as the mild, moderate and severe degree of a handicap when the scores are between 0 to 30,31 to 60 and 61 to 100 points, respectively [27].

Visual analogue scale (VAS) was used to measure the intensity of the symptoms. For VAS assessment, the patients were requested to rate the severity of their symptoms on a vertical $10-\mathrm{cm}$ line. One end of the line was anchored with the statement "I feel perfectly steady" and the other end by "worst possible unsteadiness."

The DHI and VAS scales were evaluated before VRT intervention and at "one-week", "two-week" and "four-week" post-rehabilitation intervals. 


\section{Statistical Analysis}

The data analyses were carried out with a statistical package of SPSS (SPSS Inc. Chicago, Illinois, USA, Version 21). Descriptive statistics were used to characterize the study population (mean and standard deviation, and percentage). Independent t-tests were used to compare continuous variables and Chi-square tests for categorical variables. Generalized estimating equations (GEE) regression analyses with robust standard errors were performed to identify potential predictors for the longitudinal alternation in $\mathrm{DHI}$ score per therapy session. The significance level was set at 0.05 . All quantitative data are presented as mean ( \pm Standard deviation).

\section{RESULTS}

Table 1 summarizes the demographic and baseline clinical characteristics of the subjects. Thirty-one patients with UVH met the inclusion criteria (16 males; 15 females) and entered into the study. The average age of participants was $39.48( \pm 10.96)$ years (range: 24-60 years). The mean vestibular asymmetry was $44.06 \%( \pm 8.31)$, and the meantime from the onset was $4.58( \pm 2.28)$ years.

Table 1: Demographic Characteristics of the Participants

\begin{tabular}{|c|c|c|}
\hline Characteristics & \multicolumn{2}{|c|}{$\mathrm{n}(\%)$ or mean } \\
\hline \hline Age (year) & \multicolumn{2}{|c|}{$39.48( \pm 10.96)$} \\
\hline Duration of symptoms (year) & \multicolumn{2}{|c|}{$4.58( \pm 2.28)$} \\
\hline Degree of hearing loss & Normal & $8(25.8 \%)$ \\
& Slight & $10(32.3 \%)$ \\
& Mild & $8(25.8 \%)$ \\
& Moderate & $5(16.1 \%)$ \\
\hline Gender & Male & $16(61.6 \%)$ \\
& Female & $15(48.4 \%)$ \\
\hline
\end{tabular}

For the primary outcome, DHI, the GEE analysis indicated a significant main effect of Time $(p<0.001)$.
The mean total $\mathrm{DHI}$ score significantly decreased by 15 points after one week, 24 points after two weeks and 15 points after four weeks of the last VRT session. The current evidence suggests that a change of $>18$ points in $\mathrm{DHI}$ scale is defined as the minimal clinically significant treatment for the vestibular function) (Table 2).

Figure 1 demonstrates the total $\mathrm{DHI}$ values at different times of assessments. An ANOVA analysis showed a significant reduction and a consequent improvement in $\mathrm{DHI}$ scores after vestibular exercises in all domains: emotional, physical and functional $(p<0.001)$.

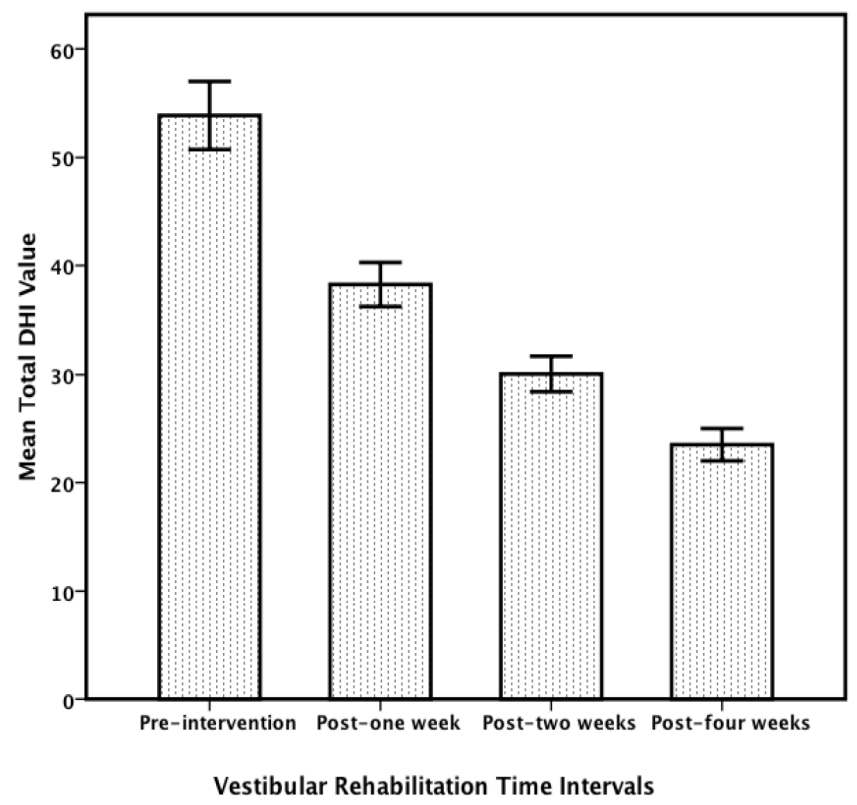

Figure 1: Comparison of total $\mathrm{DHI}$ values following VRT across different time-points.

The GEE analysis showed that the degree of hearing loss $(p=0.003)$ had a significant impact on the VRT outcomes. However, the effect of gender $(p=0.618)$, age $(p=0.164)$, nor vestibular symptom's duration $(p=0.935)$ were not statistically significant on VRT exercise outcomes (Table 3).

Table 2: Comparison of the Mean Dizziness Handicap Inventory (DHI) Scores at Different Time Points of Assessments. The Data are Presented as Mean (士SD)

\begin{tabular}{|c|c|c|c|c|}
\hline \multirow{2}{*}{ Parameter } & \multicolumn{4}{|c|}{ Vestibular rehabilitation phase } \\
\cline { 2 - 5 } & Pre-VRT & Post 1-week VRT & Post 2-week VRT & Post 4-week VRT \\
\hline \hline DHI-physical & $16.13( \pm 3.61)$ & $11.81( \pm 2.79)$ & $8.84( \pm 2.67)$ & $6.97( \pm 1.99)$ \\
\hline DHI-emotional & $17.42( \pm 4.48)$ & $11.55( \pm 2.51)$ & $9.35( \pm 2.15)$ & $6.52( \pm 1.93)$ \\
\hline DHI-functional & $20.32( \pm 3.90)$ & $14.90( \pm 3.30)$ & $12.0( \pm 2.63)$ & $10.05( \pm 3.11)$ \\
\hline DHI-total & $53.87( \pm 8.53)$ & $38.26( \pm 5.51)$ & $30.06( \pm 4.43)$ & $23.84( \pm 4.06)$ \\
\hline
\end{tabular}


Table 3: Generalized Estimating Equations Model to Assess the Effects of Predictor Variables on Dizziness Handicap Inventory (DHI) Score

\begin{tabular}{|c|c|c|c|c|c|}
\hline \multicolumn{2}{|c|}{ Variable } & \multirow{2}{*}{$\begin{array}{c}\text { Estimate } \\
55.796\end{array}$} & \multirow{2}{*}{$\begin{array}{c}\text { Standard Error } \\
4.123\end{array}$} & \multirow{2}{*}{$\frac{\mathbf{Z}}{13.533}$} & \multirow{2}{*}{$\begin{array}{c}\text { p-value } \\
<0.001\end{array}$} \\
\hline Intercept & & & & & \\
\hline Age & & 0.078 & 0.056 & 1.393 & 0.164 \\
\hline \multirow{4}{*}{$\begin{array}{l}\text { Degree of } \\
\text { hearing loss } \\
(\mathrm{HL})\end{array}$} & \multirow{4}{*}{$\begin{array}{c}\text { Normal }^{*} \\
\text { Slight HL } \\
\text { Mild HL } \\
\text { Moderate HL }\end{array}$} & -- & --- & --- & --- \\
\hline & & -0.927 & 1.727 & -0.537 & 0.591 \\
\hline & & 1.975 & 1.898 & 1.041 & 0.298 \\
\hline & & 7.861 & 2.587 & 3.039 & 0.002 \\
\hline Duration & & -0.023 & 0.282 & -0.082 & 0.935 \\
\hline Time & & -9.935 & 0.468 & -21.229 & $<0.001$ \\
\hline \multirow[t]{2}{*}{ Gender } & Male* $^{*}$ & -- & -- & -- & -- \\
\hline & Female & 0.678 & 1.395 & 0.499 & 0.618 \\
\hline
\end{tabular}

*: Reference Category.

Table 4: Comparison of the Mean VAS Scores at Different Time Points of Study

\begin{tabular}{|c|c|c|c|c|}
\hline \multirow{2}{*}{ Parameter } & \multicolumn{4}{|c|}{ Vestibular rehabilitation phase } \\
\cline { 2 - 5 } & Pre-VRT & Post 1-week VRT & Post 2-week VRT & Post 4-week VRT \\
\hline \hline VAS score & $7.71( \pm 0.92)$ & $4.81( \pm 0.73)$ & $3.77( \pm 0.61)$ & $3.16( \pm 0.97)$ \\
\hline
\end{tabular}

Table 5: Generalized Estimating Equations (GEE) Model to Assess the Effects of Predictor Variables on the Visual Analogue Scale (VAS) Values

\begin{tabular}{|c|c|c|c|c|c|}
\hline \multicolumn{2}{|c|}{ Variable } & \multirow{2}{*}{$\begin{array}{c}\text { Estimate } \\
8.516\end{array}$} & \multirow{2}{*}{$\begin{array}{c}\text { Standard Error } \\
0.543\end{array}$} & \multirow{2}{*}{$\begin{array}{c}\mathbf{Z} \\
15.772\end{array}$} & \multirow{2}{*}{$\begin{array}{c}\text { p-value } \\
<0.001\end{array}$} \\
\hline Intercept & & & & & \\
\hline Age & & 0.009 & 0.011 & 0.818 & 0.413 \\
\hline \multirow{4}{*}{$\begin{array}{l}\text { Degree of } \\
\text { hearing loss } \\
(H L)\end{array}$} & \multirow{4}{*}{$\begin{array}{c}\text { Normal* }^{*} \\
\text { Slight HL } \\
\text { Mild HL } \\
\text { Moderate HL }\end{array}$} & --- & --- & --- & --- \\
\hline & & -0.09 & 0.256 & -0.532 & 0.725 \\
\hline & & -0.197 & 0.287 & -0.686 & 0.492 \\
\hline & & 0.073 & 0.407 & 0.179 & 0.858 \\
\hline Duration & & -0.001 & 0.043 & -0.023 & 0.981 \\
\hline Time & & -1.468 & 0.062 & -23.677 & $<0.001$ \\
\hline \multirow[t]{2}{*}{ Gender } & Male* $^{*}$ & --- & --- & --- & --- \\
\hline & Female & -0.178 & 0.233 & -0.764 & 0.445 \\
\hline
\end{tabular}

The mean baseline VAS score was $7.71( \pm 0.91)$ that significantly decreased at one, two, and four weeks post-intervention to $4.81( \pm 0.73), 3.77( \pm 0.61)$, and 3.16 $( \pm 0.97)$, respectively (Table 4$)$.

In terms of VAS values, GEE analysis showed that the effect of degree of hearing loss ( $p>0.05)$, gender $(p=0.45)$, age $(p=0.413)$, and vestibular symptom's duration $(p=0.98)$ on therapy outcomes were not statistically significant (Table $\mathbf{5}$ ).

\section{DISCUSSION}

The results of the current study indicated that a customized VRT could significantly improve the dizziness-related disability in a majority of the patients with chronic UVH. Our findings showed a significant reduction in $\mathrm{DHI}$ scores compared with the baseline values that persisted for 4 weeks post-intervention. In the initial visit, $71 \%(22 / 31)$ of the patients experienced moderate or severe dizziness handicap. However, only 
$19 \%(6 / 31)$ of individuals showed a moderate degree of disability at the end of the VRT course. These findings are inconsistent with Topus et al. [28] findings which indicated a remarkable improvement of symptoms $(77 \%)$ in patients with chronic UVD, while in $23 \%$ of cases no effective changes were observed.

Similarly, Verdecchia et al. [29] reported that VRT significantly reduced the baseline mean $\mathrm{DHI}$ score (40 to 24 points) in patients with chronic UVH.

We also observed an improvement in the severity of symptoms, as assessed by the VAS, following rehabilitation exercises. Although the baseline VAS score was rather high (mean: $7.71( \pm 0.91)$ ), it decreased significantly following the VRT.

It has been demonstrated that UVH made significant changes in neural activity patterns of the vestibular nuclei on either side of the midline of the brainstem. It also influences the transmission of vestibular activity to the cerebellum and the reticular formation areas. In such situations, neuronal inputs arriving from the vestibular apparatus to ipsilateral vestibular nuclei will be decreased compared to that reaching to the contralateral nuclei. Therefore, the brain interprets the discrepancy between two incoming firing rates as a head rotation toward the contralateral side [30-32]. This may lead to a debilitating series of symptoms, including dizziness, vertigo, nausea, oscillopsia, distortions in the perception of body orientation and movement, postural instability, gait ataxia, or falling toward the affected side [1-3, 33, 34].

Vestibular rehabilitation exercises have been designed to accelerate and improve central nervous system compensation process following vestibular disorders. During the compensation process, the altered pattern of neural activity proceeds towards normal condition, and the symptoms of the UVH will be diminished. Although the majority of our study population recovered rapidly over time and lost most of their adverse symptoms following VRT exercises, the speed of symptom's recovery showed significant between-subject variations [35]. Interestingly, some patients showed incomplete recovery process so that they continue to experience their unfavourable complaints even at the end of the VRT course. The reason for this poor recovery is not fully understood yet; however, some studies have concluded that there might be a critical period for the establishment of vestibular compensation [36, 37]. The UVH itself may inadvertently generate situations for chronic vestibular disorders. It seems that if the UVH procedure leaves some vestibular fibres intact and functional, the neural signals from these fibres may interfere with the vestibular compensation process [34].

In this study, we utilized a combination of habituation and adaptation exercises for the treatment of UVH. The efficacy of adaptation exercises has been indicated in patients with normal vestibular function and those with UVH. The adaptation interventions are based on the demonstrated ability of the vestibular system to modify the magnitude of the vestibule-ocular reflex in response to a head movement [38, 39]. The habituation exercises are based on this hypothesis that repeated exposure to a provocative stimulus will reduce the motion-provoked symptoms. Clinical evidence shows that the habituation exercises could result in long-term alternations within the nervous system $[38,40]$.

Several factors might have contributed to a patient's recovery following VRT. Results from our study showed that age did not affect the VRT outcomes. However, at discharge, older patients walked more slowly than younger patients. Furthermore, we did not find a relationship between the gender variable and $\mathrm{DHI}$ and VAS values. These results are inconsistent with the findings of Herman et al. [41] that reported specific patient characteristics, such as gender and age, were not correlated to any measured outcomes.

\section{CONCLUSION}

The findings of this study indicated that four weeks of VRT could be an effective treatment option for complicated patients with UVH. The VRT exercises significantly improved the balance and postural stability and decreased the self-reported scores of the $\mathrm{DHI}$ scale.

\section{CONFLICT OF INTEREST DISCLOSURES}

The authors declare no potential conflicts of interest.

\section{ACKNOWLEDGEMENTS}

This study was the result of the M.S. thesis registered in Ahvaz Jundishapur University of Medical Sciences (AJUMS), Ahvaz, Iran and financially supported by the Vice-Chancellor for Research of the AJUMS (Grant number: U-97142). 


\section{REFERENCES}

[1] Rossi-Izquierdo M, Santos-Perez S, Soto-Varela A. What is the most effective vestibular rehabilitation technique in patients with unilateral peripheral vestibular disorders? Eur Arch Otorhinolaryngol 2011; 268: 1569-74. https://doi.org/10.1007/s00405-011-1532-z

Gabilan YP, Perracini MR, Munhoz MS, Gananc FF. Aquatic physiotherapy for vestibular rehabilitation in patients with unilateral vestibular hypofunction: exploratory prospective study. J Vestib Res 2008; 18: 139-46.

[3] Brodovsky JR, Vnenchak MJ. Vestibular rehabilitation for unilateral peripheral vestibular dysfunction. Physical Ther 2013; 93: 293-96.

https://doi.org/10.2522/ptj.20120057

[4] Wei SH, Chen PY, Chen HJ, Kao CL, Schubert MC. Visual afference mediates head and trunk stability in vestibular hypofunction. J Clin Neurosci 2016; 29: 139-44. https://doi.org/10.1016/j.jocn.2015.10.037

McDonnell M, Hillier SL. Vestibular rehabilitation for unilateral peripheral vestibular dysfunction. Cochrane Database Syst Rev 2015; 1: CD005397.

https://doi.org/10.1002/14651858.CD005397.pub4

[6] Arthur JC, Kortte KB, Shelhamer M, Schubert MC. Linear path integration deficits in patients with abnormal vestibular afference. Seeing Perceiving 2012; 25: 155-78. https://doi.org/10.1163/187847612X629928

[7] Han BI, Song HS, Kim JS. Vestibular rehabilitation therapy: review of indications, mechanisms, and key exercises. J Clin Neurol 2011; 7: 184-96.

https://doi.org/10.3988/jen.2011.7.4.184

[8] Martins E, Silva DC, Bastos VH, de Oliveira Sanchez M, Nunes MK, Orsini M, et al. Effects of vestibular rehabilitation in the elderly: a systematic review. Aging Clin Exp Res 2016; 28: 599-606. https://doi.org/10.1007/s40520-015-0479-0

[9] Jafarzadeh S, Pourbakht A, Bahrami E, Jalaie S, Bayat A. Effect of early vestibular rehabilitation on vertigo and unsteadiness in patients with acute and sub-acute head trauma. Iran J Otorhinolaryngol 2018; 30(2): 85-90.

[10] Rossi-Izquierdo M, Santos-Perez S, Soto-Varela A. What is the most effective vestibular rehabilitation technique in patients with unilateral peripheral vestibular disorders? Eur Arch Otorhinolaryngol 2011; 268: 1569-74. https://doi.org/10.1007/s00405-011-1532-z

[11] Şahin E, Dinç ME, Yayla Özker B, Çöpürgensli C, Konaklığlu M, Özçelik T. The value of vestibular rehabilitation in patients with bilateral vestibular dysfunction. J Int Adv Otol 2017; 13(3): 385-389. https://doi.org/10.5152/iao.2017.3856

[12] Herdman SJ. Vestibular rehabilitation. Curr Opin Neurol 2013; 26(1): 96-101. https://doi.org/10.1097/WCO.0b013e32835c5ec4

[13] Hillier S, McDonnell M. Vestibular rehabilitation effective in improving dizziness and function after unilateral peripheral vestibular hypofunction? An abridged version of a Cochrane Review. Eur J Phys Rehabil Med 2016; 52 (4): 541-56.

[14] Kundakci B, Sultana A, Taylor AJ, Alshehri MA. The effectiveness of exercise-based vestibular rehabilitation in adult patients with chronic dizziness: a systematic review. F1000 Res 2018; 7: 276.

https://doi.org/10.12688/f1000research.14089.1

[15] Gandolfi MM, Reilly EK, Galatioto J, Judson RB, Kim AH. Cost-effective analysis of unilateral vestibular weakness investigation. Otol Neurotol 2015; 36(2): 277-281. https://doi.org/10.1097/MAO.0000000000000649

[16] Lucieer F, Vonk P, Guinand N, Stokroos R, Kingma H, van de Berg R. Bilateral vestibular hypofunction: insights in etiologies, clinical subtypes, and diagnostics. Front Neurol 2016; 7: 26

https://doi.org/10.3389/fneur.2016.00026

[17] Bayat A, Saki A. Effects of vestibular rehabilitation interventions in the elderly with chronic unilateral vestibular hypofunction. Iran J Otorhinolaryngol 2017; 29(4): 183-88.

[18] Voelker CC, Lucisano A, Kallogjeri D, Sinks BC, Goebel JA Comparison of the gaze stabilization test and the dynamic visual acuity test in unilateral vestibular loss patients and controls. Otol Neurotol 2015; 36(4): 746-53. https://doi.org/10.1097/MAO.0000000000000689

[19] Whitney SL, Marchetti GF. Management of the elderly person with vestibular hypofunction. In: Herdman SJ (editor). Vestibular rehabilitation. F.A. Davis Company, Philadelphia 2007; pp. 376-90.

[20] Herdman SJ, Hall CD, Maloney B, Knight S, Ebert M, Lowe $\mathrm{J}$. Variables associated with outcome in patients with bilateral vestibular hypofunction: preliminary study. J Vestib Res 2015; 25(3-4): 185-94.

https://doi.org/10.3233/VES-150556

[21] Hall CD, Herdman SJ, Whitney SL, Cass SP, Clendaniel RA, Fife TD. Vestibular rehabilitation for peripheral vestibular hypofunction: an evidence-based clinical practice guideline. $\mathrm{J}$ Neurol Phys Ther 2016; 40(2): 124-55. https://doi.org/10.1097/NPT.0000000000000120

[22] Bayat A, Pourbakht A, Saki N, Zainun Z, Nikakhlagh S, Mirmomeni G. Vestibular rehabilitation outcomes in the elderly with chronic vestibular dysfunction. Iran Red Crescent Med J 2012; 14(11): 705-8. https://doi.org/10.5812/ircmi.3507

[23] Whitney SL, Alghwiri AA, Alghadir A. An overview of vestibular rehabilitation. Hand Clin Neurol 2016; 137: 187205.

https://doi.org/10.1016/B978-0-444-63437-5.00013-3

[24] Giray M, Kirazli Y, Karapolat H, Celebisoy N, Bilgen C, Kirazli $\mathrm{T}$. Short-term effects of vestibular rehabilitation in patients with chronic unilateral vestibular dysfunction: a randomized controlled study. Arch Phys Med Rehab 2009; 90(8): 132531.

https://doi.org/10.1016/j.apmr.2009.01.032

[25] Chubert MC, Migliaccio AA, Clendaniel RA, Allak A, Carey JP. Mechanism of dynamic visual acuity recovery with vestibular rehabilitation. Arch Phys Med Rehabil 2008; 89: 500-7.

https://doi.org/10.1016/j.apmr.2007.11.010

[26] Jacobson G, Newman C. The development of the dizziness handicap inventory. Arch Otolaryngol Head Neck Surg 1990; 116: 424-27. https://doi.org/10.1001/archotol.1990.01870040046011

[27] Whitney SL, Wrisley DM, Brown KE, Furman JM. Is perception of handicap related to functional performance in persons with vestibular dysfunction? Otol Neurotol 2004; 25 139-43.

https://doi.org/10.1097/00129492-200403000-00010

[28] Topuz O, Topuz B, Ardiç FN, Sarhuş M, Ogmen G, Ardiç F. Efficacy of vestibular rehabilitation on chronic unilateral vestibular dysfunction. Clin Rehabil 2004; 18(1): 76-83. https://doi.org/10.1191/0269215504cr704oa

[29] Verdecchia DH, Mendoza M, Sanguineti F, Binetti AC Outcomes after vestibular rehabilitation and $\mathrm{Wii} B$ therapy in patients with chronic unilateral vestibular hypofunction. Acta Otorrinolaringol Esp 2014; 65(6): 339-45. https://doi.org/10.1016/i.otorri.2014.02.012

[30] Fetter M, Dichgans J. Adaptive mechanisms of VOR compensation after unilateral peripheral vestibular lesions in humans. J Vestib Res 1990; 1: 9-22.

[31] Schubert MC, Minor LB. Vestibulo-ocular physiology underlying vestibular hypofunction. Phys Ther 2004; 84(4): 373-85. 
[32] Herdman SJ, Hall CD, Schubert MC, Das VE, Tusa RJ. Recovery of dynamic visual acuity in bilateral vestibular hypofunction. Arch Otolaryngol Head Neck Surg 2007; 133: 383-89.

https://doi.org/10.1001/archotol.133.4.383

[33] Binetti AC, Varela AX, Lucarelli DL, Verdecchia DH. Unilateral head impulses training in uncompensated vestibular hypofunction. Case Rep Otolaryngol 2017; 2017: 2145173.

https://doi.org/10.1155/2017/2145173

[34] Horn ER. Critical periods in vestibular development or adaptation of gravity sensory systems to altered gravitational conditions? Arch Ital Biol 2004; 142(3): 155-74

[35] Herdman SJ, Clendaniel RA. Physical therapy management of bilateral vestibular hypofunction and Loss. In: Herdman SJ, Clendaniel RA, editors. Vestibular rehabilitation. $4^{\text {th }}$ edition. Philadelphia: Davis Company 2014; pp. 432-50.

[36] Newlands SD, Dara S, Kaufman GD. Relationship of static and dynamic mechanisms in vestibuloocular reflex compensation. Laryngoscope 2005; 115: 191-204.

https://doi.org/10.1097/01.mlg.0000154718.80594.2e
[37] Beraneck M, McKee JL, Aleisa M, Cullen KE. Asymmetric recovery in cerebellar-deficient mice following unilateral labyrinthectomy. J Neurophysiol 2008; 100(2): 945-58. https://doi.org/10.1152/jn.90319.2008

[38] Clendaniel RA. The effects of habituation and gaze-stability exercises in the treatment of unilateral vestibular hypofunction - preliminary results. J Neurol Phys Ther 2010; 34(2): 111-16. https://doi.org/10.1097/NPT.0b013e3181deca01

[39] Szturm T, Ireland DJ, Lessing-Turner M. Comparison of different exercise programs in the rehabilitation of patients with chronic peripheral vestibular dysfunction. J Vest Res 1994; 4(6): 461-79.

[40] Telian SA, Shepard NT, Smith-Wheelock M, Kemink JL. Habituation therapy for chronic vestibular dysfunction: preliminary results. Otolaryngol Head Neck Surg 1990; 103(1): 89-95.

https://doi.org/10.1177/019459989010300113

[41] Herdman SJ, Hall CD, Delaune W. Variables associated with outcome in patients with unilateral vestibular hypofunction. Neurorehabil Neural Repair 2012; 26(2): 151-62. https://doi.org/10.1177/1545968311407514

DOI: https://doi.org/10.6000/2292-2598.2019.07.03.6

(C) 2019 Angali et al.; Licensee Lifescience Global.

This is an open access article licensed under the terms of the Creative Commons Attribution Non-Commercial License (http://creativecommons.org/licenses/by-nc/3.0/) which permits unrestricted, non-commercial use, distribution and reproduction in any medium, provided the work is properly cited. 